\title{
Chemical characterization and in vitro evaluation of birch sap and a complex of plant extracts for potential use in cosmetic anti-ageing products
}

\author{
Mārtiņš Borodušḳis ${ }^{1 *}$, Elza Kaktiņa ${ }^{1}$, Ilze Blāḳe ${ }^{1}$, Ilva Nakurte², Diana Dzabijeva², \\ Iveta Kusiṇa ${ }^{3}$, Santa Mičule ${ }^{3}$, Anna Ramata-Stunda' \\ ${ }^{1}$ University of Latvia, Faculty of Biology, Department of Microbiology and Biotechnology, Jelgavas 1, Riga LV-1004, Latvia \\ ${ }^{2}$ University of Latvia, Faculty of Chemistry, Jelgavas 1, Riga LV-1004, Latvia \\ ${ }^{3}$ MADARA Cosmetics JSC, Zeltiņu 131, Marupe LV-2167, Latvia \\ *Corresponding author, E-mail: martins.boroduskis@lu.lv
}

\begin{abstract}
Cosmetic manufacturers are continuously searching for new active ingredients to satisfy consumer needs. This leads to growing demand of botanical extracts with characterized chemical composition and proven efficacy. The aim of the study was to characterize the chemical composition of birch sap and a complex of seven plant extracts, with brand name Cell Repair Bio-Complex (CRB), and to evaluate their effects on skin cells. HPLC analysis was used to identify dominating compounds. Effect on proliferation of dermal fibroblasts and keratinocytes was assessed using the IncuCyte ZOOM system. Results show that flavonoid and phenolic acid rich CRB possesses antiradical activity and stimulates dermal fibroblast proliferation at low concentration. Birch sap stimulates both dermal fibroblasts and keratinocytes at high concentrations. Stimulatory effect of keratinocytes was observed also for the combination of birch sap and $\mathrm{CRB}$. The findings indicate the potential of $\mathrm{CRB}$ to be used as an active ingredient, whereas birch sap used at high concentrations could substitute water base and add additional efficacy.
\end{abstract}

Key words: birch sap, dermal fibroblasts, epidermal keratinocytes, plant extracts, skin antiageing.

Abbreviations: ARA, antiradical activity; CRB, Cell Repair Bio-Complex (a brand name for a combination of seven plant extracts for cosmetic use); DPPH, 2.2- diphenyl-1-picrylhydrazyl radical; ROS, reactive oxygen species.

\section{Introduction}

Skin aging can be classified into intrinsic aging, which is mainly attributed to genetic factors and alterations of the endocrine environment leading to reduced structural integrity and loss of physiological function, and extrinsic aging caused by direct contact with environmental factors (Fisher et al. 2002; Makrantonaki, Zouboulis 2007). Studies have shown that intrinsic or chronological skin ageing contributes to reduced amount of elastin (El-Domyati et al. 2002), lower production of collagen, decreased density and senescence of dermal fibroblasts and reduced fibroblast attachment to collagen fibers (Takeda et al. 1992; Varani et al. 2006). All the above-mentioned factors and processes are responsible for the thinning of skin and decrease of elasticity (Varani et al. 2006; El-Domyati et al. 2012). Apart from those, changes in pigmentation are also a natural indication of intrinsic skin ageing (Yaar, Gilchrest 2001).

In addition to internal factors that lead to the natural pathway of aging, several environmental factors contribute to this process and accelerate the onset of aging in skin (Zouboulis, Makarantonaki 2011). One of the main external contributors to skin aging is chronic exposure to low-grade UV radiation and the aging process accordingly named photoaging. Photoaged skin is mostly characterized by hyperpigmentation spots (Yaar, Gilchrest 2001; Nichols, Katiyar 2010), coarse wrinkling, decreased elasticity, laxity, a yellow hue (El-Domyati et al. 2002; Lewis et al. 2004; Trojahn et al. 2015), increased fragility and lower wound healing capacity (Weiss et al. 1988b; Cao et al. 2008). Studies have shown that sun-exposure induces elastin gene transcriptional activity leading to morphologically abnormal elastin accumulation in comparison with protected skin (Bernstein et al. 1996; El-Domyati et al. 2002). Sun-exposed skin areas are subject to both UV-A (400 to $315 \mathrm{~nm}$ ) and UV-B (315 to $280 \mathrm{~nm}$ ) irradiation, with UV-A being the dominant source of damage. Studies using mice model have shown that UV-A is deeply penetrating and causes damage in the dermal layer, while UV-B penetrates epidermis causing direct DNA damages and inducing inflammation (Kligman et al. 1985).

The main cellular and tissue level changes during photoaging are proliferation and adhesion impairment of keratinocytes (Bosset et al. 2003) as well as fragmented and disorganized collagen fibers (Bernstein et al.1996) in dermis and UV-B-induced upregulation of collagen degrading 
matrix metalloproteinases (Brenneisen et al. 2000). Various studies have shown that UV radiation-induced accumulation of reactive oxygen species (ROS) in skin tissue is the underlying mechanism of photoaging (Rhie et al. 2001), although smoking, pollution and endogenous metabolic processes are also linked to the production and accumulation of ROS in skin aging (Guinot et al. 2002; Trojahn et al. 2015).

Increasing life expectancy along with public pressure to retain youthful appearance has led to increased demand for efficient skin antiaging products. The accumulated knowledge on mechanisms of skin aging allows to develop better and more effective skin care products to slow down aging and protect skin from undesired exogenous effects.

Plants are thought of as valuable sources of natural organic cosmetic ingredients possessing many valuable properties for maintenance and improvement of skin health. Plant-specific active substances with antioxidant, anti-inflammatory, anti-mutagenic, anti-carcinogenic and immunomodulatory properties have the ability to exert striking inhibitory effects on diverse cellular and molecular events and therefore are gaining considerable attention for the prevention of exogenous skin damage (Afaq et al. 2009; Duque et al. 2017). Today, plant antioxidants, particularly polyphenols, are among the most sought cosmetic ingredients (Nichols, Katiyar 2010; Mukherjee et al. 2011). It has been shown that polyphenols and flavonoids of green tea possess protective activity against UV-induced skin damage due to their antioxidative, anti-inflammatory and DNA repair activities (Kim et al. 2001; Vayalil et al. 2004). To reverse signs of aging use of plant derived ingredients with characterized skin cell proliferation promoting properties is increasing (Moore et al. 2006; Masaki 2010; Gopaul et al. 2012). Replacing water with plant juices and infusions could also be a growing trend in near future to further boost product efficacy. Birch sap, for instance, contains vitamin $\mathrm{C}$ as well as various phenolic compounds and glycosides, which in previous studies have shown antiradical activity and a great potential as an effective water replacement (Kūka et al. 2013; Mukherjee et al. 2011).

In this study we chemically characterized composition of plant ethanolic extracts and birch sap and analyzed their antiradical and skin cell stimulating properties to evaluate the potential for their inclusion in antiaging skin care products.

\section{Materials and methods}

\section{Obtaining of test material}

Birch sap from Betula pendula was collected during the sap exudation period in the middle of April 2016. Sap was collected in the Latvia (Salaspils district). Birch sap was delivered frozen to University of Latvia by MADARA Cosmetics JSC. Sap was immediately aliquoted $20 \mathrm{~mL}$ per vial, freeze-dried and dissolved in $1 \mathrm{~mL}$ sterile water to obtain 20 times concentrate.

CRB is a complex of seven plants extracts (Galium verum extract of aerial parts, Vaccinium mytillus fruit extract, Alchemilla vulgaris extract of aerial parts, Trifolium pratense flower extract, Hippophae rhamnoides fruit extract, Equisetum arvense extract of aerial parts, and Aesculus hippocastanum seed extract) was delivered to University of Latvia by MADARA Cosmetics JSC. Each of the seven individual plant extracts were produced in the extract factory "L.E.V." Ekstraktu Rūpnīca Ltd. using maceration method and 50\% ethanol/ 6\% glycerine solution as a solvent. Ratio of $1: 10 \mathrm{~m} / \mathrm{v}$ (dry plant material vs solvent) was used for extraction. The proportions of individual extracts in the CRB complex are know-how of MADARA Cosmetics JSC and were not disclosed.

\section{Antiradical activity}

Antiradical activity (ARA) was determined by DPPH assay using the high-throughput 96-well plate method as described by Herald et al. 2012. ARA were analyzed for CRB extract. The DPPH method is based on the ability to stabile free radical 2.2-diphenyl-picrylhydrasyl (DPPH) to react with hydrogen donors. The measurements of CRB extracts were done by mixing $100 \mu \mathrm{M}$ DPPH solution in ethanol with extract or standard samples. The absorbance was measured at $517 \mathrm{~nm}$, along with the blank. Based on the diagram representing the antiradical activity vs. different sample concentrations or the reference compound, the value of $\mathrm{IC}_{50}$ was determined. This value represented the sample (or the reference compound) concentration in $\mathrm{mg}$ DW needed to inhibit $50 \%$ of DPPH radicals. ARA also was expressed as ascorbic acid equivalents on extract dry weight (mg AA eq $\mathrm{mg}^{-1} \mathrm{DW}$ ), based on a calibration curve (0.03 to $0.09 \mathrm{mg} \mathrm{mL}^{-1}, R^{2}=0.998$ ). Data were obtained from three technical replicates.

\section{LC-MS analysis}

All solvents were of analytical grade. Acetonitrile and formic acid were purchased from Sigma-Aldrich (St. Louis, USA). The used deionized water (18.2 $\mathrm{M} \Omega$ ) was prepared by a Milli-Q water purification system from Millipore (Billerica, Massachusetts, USA). Chromatographic analyses were performed on a modular UHPLC system, Agilent 1290 Infinity series (Agilent Technologies, Germany). Chromatographic separation at $30^{\circ} \mathrm{C}$ using a column oven and a dC18. $2.1 \times 150 \mathrm{~mm}, 3.0 \mu \mathrm{m}$ (Waters) column for birch sap was performed and at $30^{\circ} \mathrm{C}$ using a column oven and a Kinetex C18. $2.1 \times 50 \mathrm{~mm}, 2.6 \mu \mathrm{m}$ (Phenomenex) column for CRB extract respectively. Elution solvents consisted of $0.1 \%$ formic acid in acetonitrile and $0.1 \%$ formic acid in water in gradient mode. The injection volume was $20 \mu \mathrm{L}$ birch sap concentrated 10 times. High resolution mass spectra were taken on an Agilent 6230 TOF LC/MS (Agilent Technologies, Germany) with electrospray ionization. Mass spectrometry operating conditions were 
as follows: positive ionization mode, gas temperature 325 ${ }^{\circ} \mathrm{C}$, nitrogen flow rate $12 \mathrm{~L} \mathrm{~min}^{-1}$, nebulizer pressure $45 \mathrm{psi}$, capillary voltage $4000 \mathrm{~V}$ and the applied fragmentor was 130 $\mathrm{V}$. Internal reference mass $121.050873 \mathrm{~m} / \mathrm{z}$ and 922.009798 m/z (G1969-85001 ES-TOF Reference Mass Solution Kit, Agilent Technologies \& Supelco) for all sample analyses were used. Peak detection and spectrum extraction were performed with MassHunter7.00 Software (Agilent). One full mass spectrum was acquired in profile mode, with mass range from $\mathrm{m} / \mathrm{z} 50$ to 1500 . Solutions were filtered with 0.45 $\mu \mathrm{m}$ filters (Nonpyrogenic Sterile-R, Sarstedt) to remove particulate and other suspended solid matter. The filtered samples were injected into a LC system.

\section{Cell culture and time dependent cell response profiling using the IncuCyte ZOOM system}

Immortalized human keratinocytes HaCaT (Cell Line Service) and dermal fibroblasts (obtained according to Latvian legislation) were resuspended in standard DMEM/10\%FBS (Biochrom) medium and seeded in 96well microplates (Sarstedt) at density of 3500 cells per well for $\mathrm{HaCaT}$, and 1500 cells $\mathrm{p} / \mathrm{w}$ for dermal fibroblasts and incubated in a humidified $37^{\circ} \mathrm{C}, 5 \% \mathrm{CO}_{2}$ incubator. After seeding, cells were left to adhere the surface of the plate and grow up to confluence of $50 \%$. Cell medium was drained and $100 \mu \mathrm{L}$ fresh medium as a control containing $0.5 \%(\mathrm{v} / \mathrm{v})$ solvent (50\% ethanol) and CRB extract-medium solutions in different concentrations $(0.125$ to $0.5 \% \mathrm{v} / \mathrm{v})$ were added. In the case of birch sap, fresh medium as a control containing $2.5 \%(\mathrm{v} / \mathrm{v}) 1 \times$ phosphate buffered saline and 20 times concentrated birch sap in concentrations $(0.312$ to $2.5 \% \mathrm{v} / \mathrm{v}$ ) which represented respectively 6.25 to $50 \%(\mathrm{v} / \mathrm{v})$ natural birch sap were added. Cells were imaged for $48 \mathrm{~h}$ by phase contrast imaging at magnification $100 \times$ using an IncuCyte ZOOM microscope system (Essen Biosciences).
Kinetics of cell growth were monitored using the IncuCyte integrated confluence algorithm, where confluence is a surrogate for cell number. Images were taken and recorded once per hour. Data were obtained from three biological and three technical replicates for each and represented as an increase of cell proliferation (\%), compared to the control.

\section{Statistical analysis}

All data were analyzed using GraphPad Prism 5 software and presented as mean \pm SEM. For the statistical analysis ANOVA followed by Bonferroni post-test was used to compare groups to the control. A $p$-value $<0.05$ was taken as the criterion of statistically significant difference.

\section{Results}

\section{Phytochemical analysis}

Both CRB and Birch sap extracts were analyzed using liquid chromatography high resolution mass spectrometry. The Base Peak Chromatogram in Fig. 1 shows the peak signal from the most intense mass in any given mass spectrum, plotted versus time. The phytochemical study of CRB complex led to the isolation and identification of more than sixty constituents. According to LC-ESI-TOF MS data, CRB had high content of flavonoids (60\%), phenolic acids (21\%) and amino acids (9\%). The main identified components are listed below Fig. 1. The dominating compound (represented about $14.2 \%$ of the total amount) of CRB with retention time at 19.9 min was identified as cyanidin 3-O-(3",6"-O-dimalonyl-glucoside) (11) belonging to the anthocyanins group. In total 15 different anthocyanins mainly as glycosides of anthocyanidins, such as cyanidin, delphinidin, malvinidin, petunidin and peonidin, were separated from CRB and constituted the largest amount of all separated flavonoids. The next most abundant

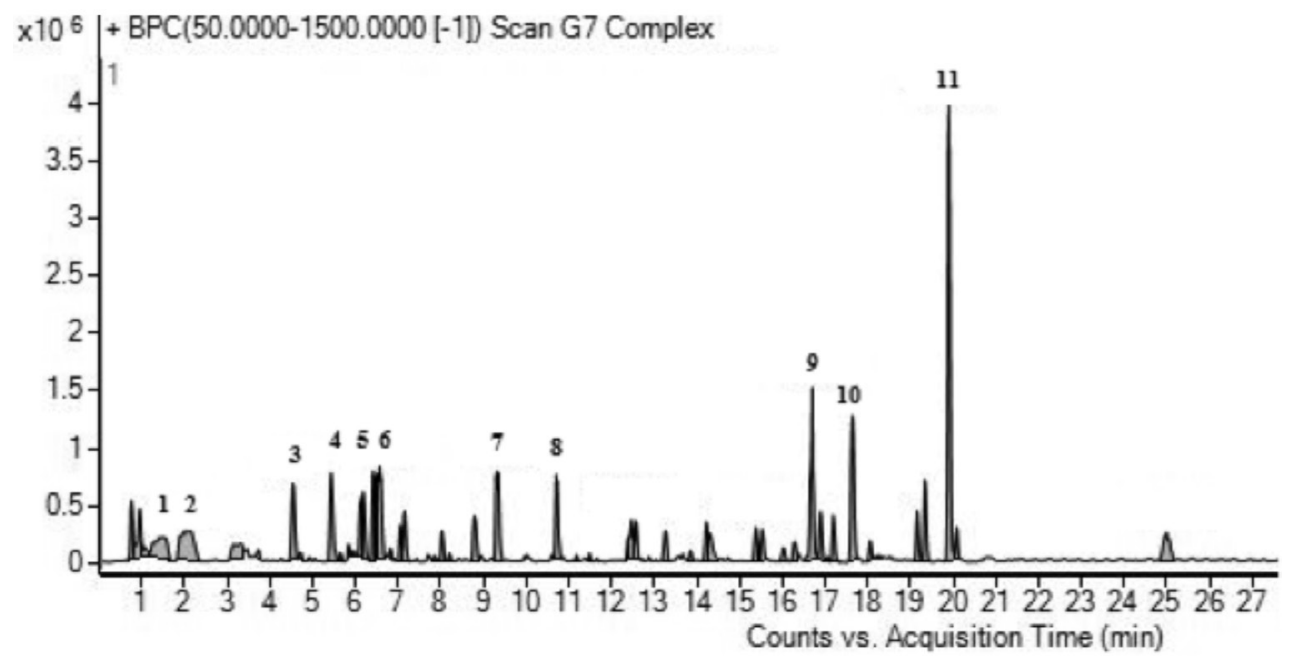

Fig. 1. Base Peak Chromatogram of the ethanolic-glycerol CRB extract. Only individual compounds with concentrations exceeding $3 \%$ are numbered. 1 , phenylalanine; 2 , caffeic acid; 3 , chlorogenic acid; 4, damascenone; 5, cyanidin 3-O-(6"-p-coumaroyl-glucoside); 6, malvidin 3-O-arabinoside; 7, formononetin; 8, glycitein; 9, pseudobaptigenin; 10, stigmastanol ferulate; 11, cyanidin 3-O-(3",6"-Odimalonyl-glucoside). 


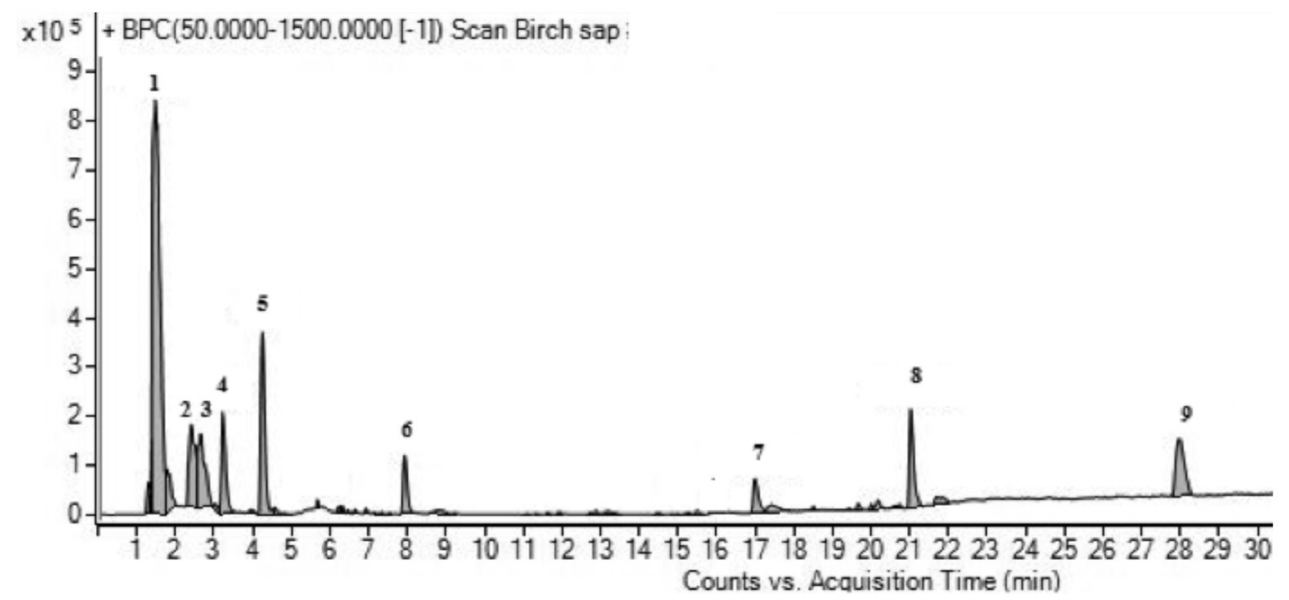

Fig. 2. Base Peak Chromatogram of the birch sap. Only individual compounds with concentrations exceeding $2 \%$ are numbered. 1 , peak of very polar birch sap fraction containing organic acids, amino acids and tyrosol 4-sulfate; 2, leucine; 3, isoleucine; 4, homovanillic acid; 5, phanylalanine; 6, (+)-catechin 3-O-glucose; 7, betulin; 8, resveratrol 3-O-glucoside; 9, unidentified tannin.

compounds were identified as stigmastanol ferulate (10), chlorogenic acid (3) and caffeic acid (2), belonging to the class of phenolic acids. Phenylalanine (1) had the highest concentration (represented about $3.5 \%$ of the total amount) of all nine amino acids separated from the CRB complex. Pseudobaptigenin, a compound typical to red clover was also identified in the CRB. Systematic phytochemical investigations should be carried out to probe the chemical profile of the separated compounds.

Fifty-five biologically active compounds were separated from birch sap. Most dominant peak with retention time at 1.49 min contained a very polar fraction of birch sap, including three organic acids, succinic acid, malic acid and citruline (represented about $6.7 \%$ of the total amount), five amino acids, and phenolic compound metabolite tyrosol 4-sulphate (represented about $32.6 \%$ of the total amount). Overall, nine amino acids were separated from birch sap and represented about $16.8 \%$ of the total amount, including leucine (2), isoleucine (3), glutamine, and phenylalanine. It should be noted that organic acids and amino acids separated from our birch sap sample are widely distributed and described in the birch sap products.
It was found that birch sap was not very rich with phenolic compounds, but it had homovanillic acid (4), (+)-catechin 3-O-glucose (6), and resveratrol 3-O-glucoside (8). Birch sap contained betulin (7) which represented about $2.4 \%$ of the total amount and some undidentified tannins.

\section{Antiradical activity}

Antiradical activity (ARA) of CRB was determined using DPPH scavenging assay. The stable free radical DPPH scavenging effect was expressed as an IC50 value, i.e. the extract concentration required to inhibit 50\% DPPH radicals. CRB reached 3.517 (mg DW) as $\mathrm{IC}_{50}$ value. The value of $\mathrm{IC}_{50}$ was compared with the reference value of vitamin $C-0.341 \mathrm{mg}$ (determined within the same experiment) and the difference was only 10.34 times. The results showed that CRB, which is a mixture of compounds, had a relatively high antiradical activity compared to pure vitamin C. ARA expressed as ascorbic acid equivalents reached 0.017 (mg AAeq per mg DW) with 0.27 RSD\%.

\section{Proliferation of skin cells in vitro}

Effect of birch sap and CRB complex on cell proliferation
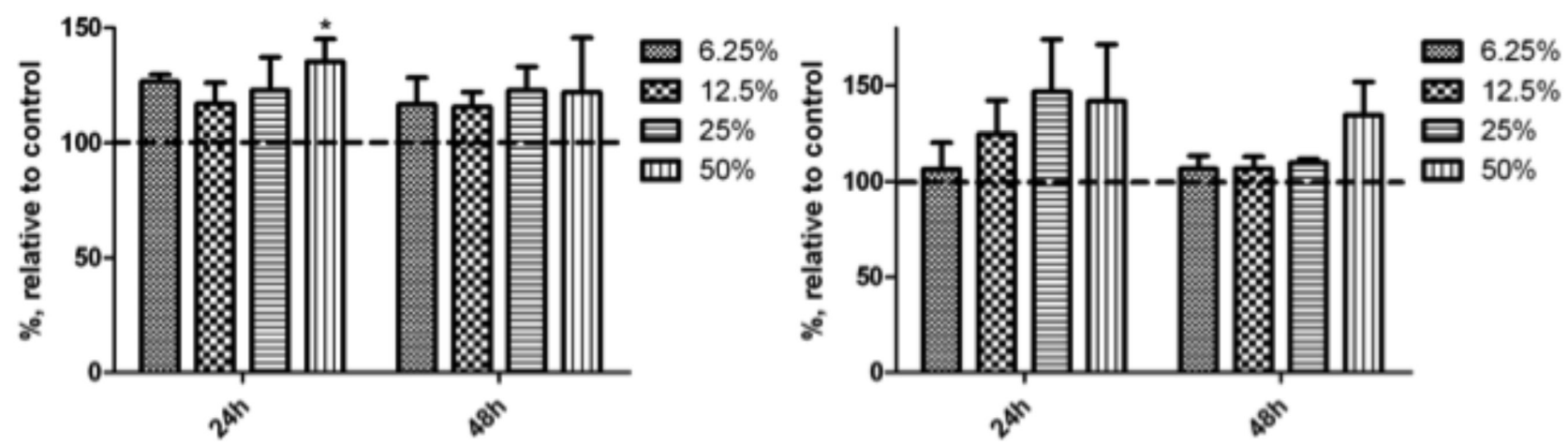

Fig. 3. Changes in proliferation of dermal fibroblasts (A) and HaCaT keratinocytes (B) in the presence of birch sap at 24 and $48 \mathrm{~h}$ of incubation. Dashed line indicates control level $\left(n=3,{ }^{\star} p<0.05\right)$. 

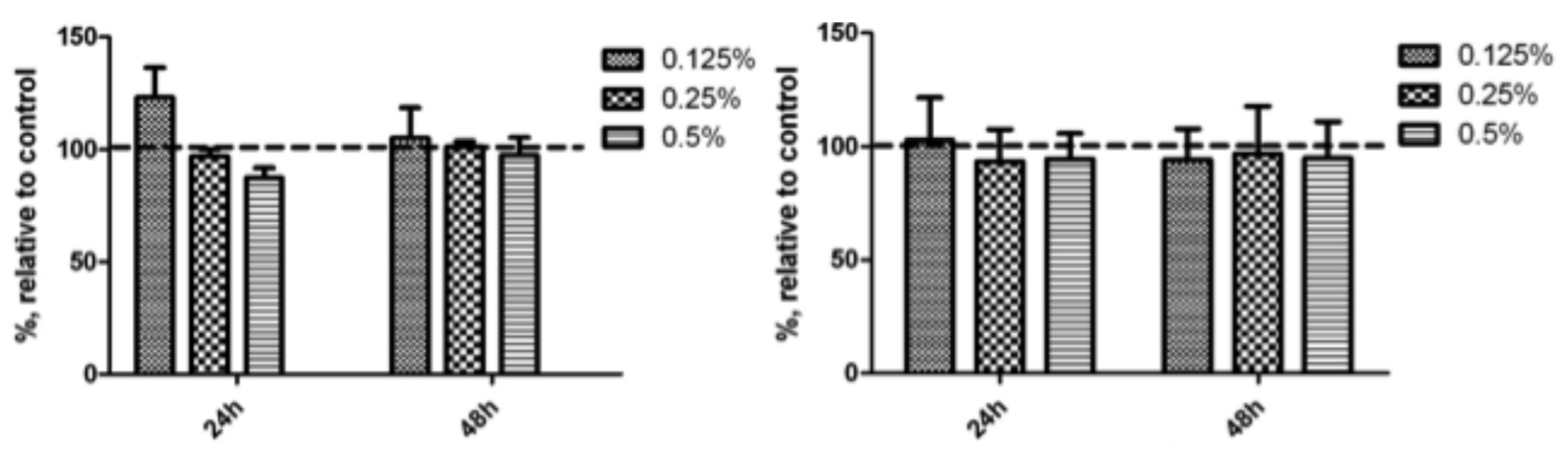

Fig. 4. Changes in proliferation of dermal fibroblasts (A) and HaCaT keratinocytes (B) in the presence of CRB complex at 24 and $48 \mathrm{~h}$ of incubation. Data are represented as relative changes in cell proliferation activity compared to control. Control level of cell proliferation $(100 \%)$ is shown with dashed line. ${ }^{\star} p$-value $<0.05, n=3$

was tested in dermal fibroblast cell cultures and $\mathrm{HaCaT}$ keratinocytes. The effects of birch sap on proliferation are shown in Fig. 3. Birch sap slightly stimulated proliferation of dermal fibroblasts during the first $24 \mathrm{~h}$ of incubation. Although all tested concentrations promoted cell proliferation, $50 \%$ birch sap showed the most stimulating activity as cell proliferation increased by $35.48 \pm 9.79 \%$. Stimulating activity remained during the $48 \mathrm{~h}$ incubation period. In $\mathrm{HaCaT}$ keratinocytes stimulation of proliferation was dose dependent, with both highest doses exhibiting the most pronounced effect. Birch sap at $50 \%$ stimulated cell proliferation by $41.75 \pm 29.70 \%$ and at $25 \%$ by $46.72 \pm$ $27.40 \%$. In a 48 -h incubation period only the highest dose showed stimulatory effect as proliferation rate increased by $34.38 \pm 17.13 \%$. Three other tested concentrations after $48 \mathrm{~h}$ showed increase in proliferation rate only by 6.52 to $9.77 \%$.

The effect of CRB complex was also tested on dermal fibroblasts and $\mathrm{HaCaT}$ keratinocytes. The results of proliferation analysis are shown in Fig. 4. CRB stimulated

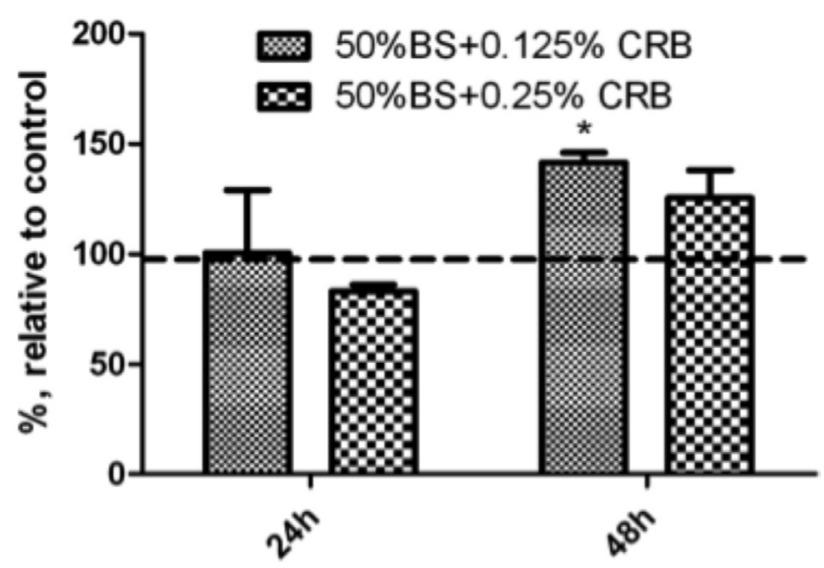

Fig. 5. Changes in proliferation of $\mathrm{HaCaT}$ keratinocytes in the presence of birch sap combination with CRB at 24 and 48 $\mathrm{h}$ of incubation. Data represented as relative changes in cell proliferation activity compared to control. Control level of cell proliferation $(100 \%)$ is shown with dashed line. ${ }^{*} p$-value $<0.05$. $n=3$. proliferation of dermal fibrobasts within the first 24 $\mathrm{h}$ of incubation at the lowest tested concentration as proliferation rate increased by $23.13 \pm 13.27 \%$. Proliferation activity decreased with increasing CRB concentrations. After $48 \mathrm{~h}$ of incubation, proliferation was at control level at all tested concentrations. No changes in proliferation of $\mathrm{HaCaT}$ keratinocytes was observed in presence of CRB.

Combination of $50 \%$ birch sap with 0.125 and $0.25 \%$ CRB showed stimulatory effect in HaCaT keratinocytes after $48 \mathrm{~h}$ of incubation as increase of $41.5 \pm 4.5 \%$ was observed in combination of sap with $0.125 \%$ CRB. Combination of birch sap with $0.25 \% \mathrm{CRB}$ promoted $\mathrm{HaCaT}$ proliferation by $25.5 \pm 12.5 \%$ (Fig. 5). Unlike birch sap and CRB alone, their combination did not have any effect on proliferation of dermal fibroblasts.

\section{Discussion}

Combinations of analytical methods are being used in evaluation of natural products to evaluate their applicability as skin care active ingredients. Data on both chemical composition and biological activity are necessary to assess potential benefits for skin. Phytochemical characterization of extracts is among the first choice of characterization methods as presence of specific compounds can clearly indicate the most probable biological effects.

In our study dominating compounds in birch sap and a complex of plant extracts (CRB) were analyzed using LChigh resolution mass spectra method. Chlorogenic acid and caffeic acid are among the dominating compounds in CRB. These compounds, along with anthocyanidins are thought to be responsible for the antioxidant properties of CRB. Chlorogenic acid has been described previously as a potent cell proliferation stimulator. Results also indicated potent wound healing capacity and potent antioxidant activity (Chen et al. 2013). Caffeic acid is known to have antiproliferative effect on HT-1080 human fibrosarcoma cell line (Rajendra Prasad et al. 2011). It is known that phenolic compounds apart from antioxidant function, have additional activities, such as stimulation of cellular 
signaling, gene expression, and modulation of enzymatic activity (Milenkovic et al. 2011).

In birch sap we found organic acids like succinic acid, malic acid and citruline, nine different amino acids some phenolic compounds, and betulin, but for these compounds effects on skin cell stimulation have not been described. Due to the limits of methods used, we did not determine the content of sugars in birch sap. From the literature, it is known that glucose and fructose constitute the major part of sugars in birch sap (Zyryanova et al. 2010). The sap is rich in mineral micro- and macroelements. Calcium $(\mathrm{Ca})$, sodium $(\mathrm{Na})$, magnesium $(\mathrm{Mg})$, potassium $(\mathrm{K})$ and iron $(\mathrm{Fe})$ are prevailing elements followed by small amounts of $\mathrm{Mn}, \mathrm{Zn}, \mathrm{Cu}, \mathrm{Al}$ and $\mathrm{Ni}$ (Zyryanova et al. 2010; Jeong et al. 2013). The concentration of glucose and fructose depends on the stage of sap exudation period. Content of sugars in fresh sap of Betula pendula is about $2 \%$ in the beginning of sap exudation and decreases in the intensive exudation period (Ozolinčius et al. 2016). It is proved that Latvian birch sap contains up to $20 \%$ more glucose and fructose than birch sap produced in Finland (Kūka et al. 2013).

Alongside chemical characterization, the effects of birch sap and combination of plant extracts on skin cells were evaluated. Our results show direct effect of CRB and concentrated birch sap on human fibroblasts and immortalized keratinocyte ( $\mathrm{HaCaT})$ cell cultures, which is similar to basic non-clinical toxicology tests performed in new biologically active compound screening. Whilst measurement of the effect of CRB and birch sap on human cell culture proliferation cannot be directly extrapolated to in vivo situations, performance of such test can provide early indication of cases where extract contains toxic substances. Also the test allows to identify beneficial stimulatory activities. Cell proliferation assays indicated the potential use of birch sap at high concentrations as a regenerating component in skin care products. CRB complex was shown to be safe to use at low concentrations, and $0.125 \%$ concentration in vitro showed stimulating activity on dermal cells. Effects of combinations of birch sap and CRB on cell proliferation allows to speculate about synergistic or antagonistic effects of the components in such combinations. Product combination stimulated proliferation in $\mathrm{HaCaT}$ keratinocytes, but use of higher CRB concentration slightly suppressed the stimulatory effect of birch sap. In dermal fibroblasts, CRB at concentration of $0.125 \%$ alone stimulated proliferation, but combination with birch sap did not have any effect on cell proliferation. Plant extracts included in the CRB have been reported in the literature to possess antioxidative, antiinflammatory and tissue regenerating properties. Species from the Galium genus have shown efficacy in reduction of oxidative stress in dermal fibroblasts (Chaher et al. 2016) and proved to be potent antiiflammatory agents (Bolivar et al. 2011). Vaccinium myrtillus extracts are reported to exert protective activity in UVA-irradiated keratinocytes (Calò, Marabini 2014). Antioxidative and anti-inflammatory properties have been reported also for Alchemilla vulgaris and Aesculus hippocastanum extracts and plants form Equisetum, Trifolium and Hippophae genera (Wilkinson, Brown 1999; Hamad et al. 2007; Stajner et al. 2009; Suryakumar, Guo et al. 2017). In general, it is difficult to know which component in CRB extract affects cell proliferation, but in this particular in vitro test system, toxic effect on cell cultures would have been observed at compound concentrations above $0.25 \% \mathrm{v} / \mathrm{v}$ in the case of CRB. Regarding birch sap, previous findings suggest that the complex of amino acids, sugars and mineral elements is the reason for promotion of cell growth and proliferation, which serves as a rationale for the inclusion of sap as an ingredient in anti-ageing cosmetic compositions.

Results of our chemical analysis and effects on stimulation of cell proliferation along with other published data on composition and biological activities of plant extracts and birch sap allows to envisage use of these natural products as cosmetic ingredients. Effects on skin cells in vitro indicate that CRB can be include in product formulations at low concentrations, whereas birch sap shows effect at high concentrations and could be used to substitute water base. A combination of birch sap and low concentration of $\mathrm{CRB}$ has stimulatory effect on keratinocytes, which indicated the high potential of this combination to be used for reversal of epidermal ageing.

\section{Acknowledgements}

This research has been funded with support of MADARA Cosmetics JSC and Pharma and Chemistry Competence Centre of Latvia.

\section{References}

Afaq F., Zaid M.A., Khan N., Dreher M., Mukhtar H. 2009. Protective effect of pomegranate-derived products on UVBmediated damage in human reconstituted skin. Exp. Dermatol. 18: 553-561.

Bernstein E.F., Chen Y.Q., Kopp J.B., Fisher L., Brown D.B., Hahn P.J., Robey F.A., Lakkakorpi J., Uitto J. 1996. Long-term sun exposure alters the collagen of the papillary dermis. Comparison of sun-protected and photoaged skin by northern analysis, immunohistochemical staining, and confocal laser scanning microscopy. J. Am. Acad. Dermatol. 34: 209-218.

Bolivar P., Cruz-Paredes C., Hernández L.R., Juárez Z.N., SánchezArreola E., Av-Gay Y., Bach H. 2011. Antimicrobial, antiinflammatory, antiparasitic, and cytotoxic activities of Galium mexicanum. J. Ethnopharmacol. 137: 141-147.

Bosset S., Bonnet-Duquennoy M., Barré P., Chalon A., Lazou K., Kurfurst R., Bonté F., Schnébert S., Disant F., Le Varlet B., Nicolas J.F. 2003. Decreased expression of keratinocyte beta1 integrins in chronically sun-exposed skin in vivo. British J. Dermatol. 148: 770-778.

Brenneisen P., Wenk J., Wlaschek M., Krieg T., ScharffetterKochanek K. 2000. Activation of p70 ribosomal protein S6 kinase is an essential step in the DNA damage-dependent 
signaling pathway responsible for the ultraviolet B-mediated increase in interstitial collagenase (MMP-1) and stromelysin-1 (MMP-3) protein levels in human dermal fibroblasts. J. Biol. Chem. 275: 4336-4344.

Calò R., Marabini L. 2014. Protective effect of Vaccinium myrtillus extract against UVA- and UVB-induced damage in a human keratinocyte cell line (HaCaT cells). J. Photochem. Photobiol. B 132: 27-35.

Cao C., Wan S., Jiang Q., Amaral A., Lu S., Hu G., Bi Z., Kouttab N., Chu W., Wan Y. 2008. All-trans retinoic acid attenuates ultraviolet radiation-induced down-regulation of aquaporin-3 and water permeability in human keratinocytes. J. Cell. Physiol. 215: 506-516.

Chaher N., Krisa S., Delaunay J.C., Bernillon S., Pedrot E., Mérillon J.M., Atmani D., Richard T. 2016. Unusual compounds from Galium mollugo and their inhibitory activities against ROS generation in human fibroblasts. J. Pharm. Biomed. Anal. 117: 79-84.

Chen W.C., Liou S.S., Tzeng T.F., Lee S.L., Liu I.M. 2013. Effect of topical application of chlorogenic acid on excision wound healing in rats. Planta Med. 79: 616-621.

Duque L., Bravo K., Osorio E. 2017. A holistic anti-aging approach applied in selected cultivated medicinal plants: A view of photoprotection of the skin by different mechanisms. Ind. Crop. Prod. 97: 431-439.

El-Domyati M., Attia S., Saleh F., Brown D., Birk D.E., Gasparro F., Ahmad H., Uitto J. 2002. Intrinsic aging vs. photoaging: a comparative histopathological, immunohistochemical, and ultrastructural study of skin. Exp. Dermatol. 11: 398-405.

Fisher G.J., Kang S., Varani J., Bata-Csorgo Z., Wan Y., Datta S., Voorhees J.J. 2002. Mechanisms of photoaging and chronological skin aging. Arch. Dermatol. 138: 1462-1470.

Gopaul R., Knaggs H.E., Lephart E.D. 2012. Biochemical investigation and gene analysis of equol: A plant and soyderived isoflavonoid with antiaging and antioxidant properties with potential human skin applications. Biofactors 38: 44-52.

Guinot C., Malvy D.J., Ambroisine L., Latreille J., Mauger E., Tenenhaus M., Morizot F., Lopez S., Le Fur I., Tschachler E. 2002. Relative contribution of intrinsic vs extrinsic factors to skin aging as determined by a validated skin age score. Arch. Dermatol. 138: 1454-1460.

Guo R., Guo X., Li T., Fu X., Liu R.H. 2017. Comparative assessment of phytochemical profiles, antioxidant and antiproliferative activities of Sea buckthorn (Hippophaë rhamnoides L.) berries. Food. Chem. 221: 997-1003.

Hamad I., Erol-Dayi O., Pekmez M., Onay-Ucar E., Arda N. 2007. Free radical scavenging activity and protective effects of Alchemilla vulgaris (L.). J. Biotechnol. 131: 40-41.

Herald T.J., Gadgil P., Tilley M. 2012. High-throughput micro plate assays for screening flavonoid content and DPPHscavenging activity in sorghum bran and flour. J. Sci. Food Agr. 92: 2326-2331.

Jeong S.J., Jeong H.S., Woo S.H., Shin C.H. 2013. Consequences of ultrafiltration and ultraviolet on the quality of white birch (Betula platyphylla var. japonica) sap during storage. Aust. J. Crop. Sci. 7: 1072-1077.

Kim J., Hwang J.S., Cho Y.K., Han Y., Jeon Y.J., Yang K.H. 2001. Protective effects of (-)-epigallocatechin-3-gallate on UVAand UVB-induced skin damage. Skin Pharmacol. Appl. Skin Physiol. 14: 11-19.

Kligman L.H., Akin F.J., Kligman A.M. 1985. The contributions of UVA and UVB to connective tissue damage in hairless mice. J.
Invest. Dermatol. 84: 272-276.

Kūka M., Čakste I., Geršebeka E. 2013. Determination of bioactive copounds and mineral substances in Latvian birch and maple saps. Proc. Latv. Acad. Sci. Sect. B 67: 437-441.

Lewis K.G., Bercovitch L., Dill S.W., Robinson-Bostom L. 2004. Acquired disorders of elastic tissue: part I. Increased elastic tissue and solar elastotic syndromes. J. Am. Acad. Dermatol. 51: $1-21$.

Makrantonaki E., Zouboulis C.C. 2007. The skin as a mirror of the aging process in the human organism--state of the art and results of the aging research in the German National Genome Research Network 2 (NGFN-2). Exp. Gerontol. 42: 879-886.

Masaki H. 2010. Role of antioxidants in the skin: Anti-aging effects. J. Dermatol. Sci. 58: 85-90.

Milenkovic D., Deval C., Dubray C., Mazur A. and Morand C. 2011. Hesperidin displays relevant role in the nutrigenomic effect of orange juice on blood leukocytes in human volunteers: a randomized controlled cross-over study. PLoS One 6: 26669.

Moore J.O., Wang Y., Stebbins W.G., Gao D., Zhou X., Phelps R., Lebwohl M., Wei H. 2006. Photoprotective effect of isoflavone genistein on ultraviolet $\mathrm{B}$-induced pyrimidine dimer formation and PCNA expression in human reconstituted skin and its implications in dermatology and prevention of cutaneous carcinogenesis. Carcinogenesis 27: 1627-1635.

Mukherjee P.K., Maity N., Nema N.K., Sarkar B.K. 2011. Bioactive compounds from natural resources against skin aging. Phytomedicine 19: 64-73.

Nichols J.A., Katiyar S.K. 2010. Skin photoprotection by natural polyphenols: Anti-inflammatory, anti-oxidant and DNA repair mechanisms. Arch. Dermatol. Res. 302: 71.

Ozolinčius R., Bareika V., Rubinskienė M., Viškelis P., Mažeika R., Staugaitis G. 2016. Chemical composition of silver birch (Betula pendula Roth.) and downy birch (Betula pubescens Ehrh.) sap. Balt. For. 22: 222-229.

Rajendra Prasad N., Karthikeyan A., Karthikeyan S., Reddy B.V. 2011. Inhibitory effect of caffeic acid on cancer cell proliferation by oxidative mechanism in human HT-1080 fibrosarcoma cell line. Mol. Cell. Biochem. 349: 11-19.

Rhie G., Shin M.H., Seo J.Y., Choi W.W., Cho K.H., Kim K.H., Park K.C., Eun H.C., Chung J.H. 2001. Aging- and photoagingdependent changes of enzymic and nonenzymic antioxidants in the epidermis and dermis of human skin in vivo. J. Invest. Dermatol. 117: 1212-1217.

Stajner D., Popović B.M., Canadanović-Brunet J., Anackov G. 2009. Exploring Equisetum arvense L., Equisetum ramosissimum L. and Equisetum telmateia L. as sources of natural antioxidants. Phytother Res. 23: 546-550.

Suryakumar G., Gupta A. 2011. Medicinal and therapeutic potential of Sea buckthorn (Hippophae rhamnoides L.). J. Ethnopharmacol. 138: 268-278.

Takeda K., Gosiewska A., Peterkofsky B. 1992. Similar, but not identical, modulation of expression of extracellular matrix components during in vitro and in vivo aging of human skin fibroblasts. J. Cell Physiol. 153: 450-459.

Trojahn C., Dobos G., Lichterfeld A., Blume-Peytavi U., Kottner J. 2015. Characterizing facial skin ageing in humans: disentangling extrinsic from intrinsic biological phenomena. Biomed Res. Int. 2015: 318586.

Varani J., Dame M.K., Rittie L., Fligiel S.E., Kang S., Fisher G.J., Voorhees J.J. 2006. Decreased collagen production in chronologically aged skin: roles of age-dependent alteration in fibroblast function and defective mechanical stimulation. 
Am. J. Pathol. 168: 1861-1868.

Vayalil P.K., Mittal A., Hara Y., Elmets C.A., Katiyar S.K. 2004. Green tea polyphenols prevent ultraviolet light-induced oxidative damage and matrix metalloproteinases expression in mouse skin. J. Invest. Dermatol. 122: 1480-1487.

Weiss J.S., Ellis C.N., Headington J.T., Voorhees J.J. 1988b. Topical tretinoin in the treatment of aging skin. J. Am. Acad. Dermatol. 19: $169-175$.

Wilkinson J.A., Brown A.M. 1999. Horse Chestnut - Aesculus hippocastanum: potential applications in cosmetic skin-care products. Int. J. Cosmetic Sci. 21: 437-447.

Yaar M., Gilchrest B.A. 2001. Ageing and photoageing of keratinocytes and melanocytes. Clin. Exp. Dermatol. 26: 583591.

Zouboulis C.C, Makrantonaki E. 2011. Clinical aspects and molecular diagnostics of skin aging. Clin. Dermatol. 29: 3-14.

Zyryanova O.A., Terazawa M., Takayoshi K., Zyryanov V.I. 2010. White birch trees as resource species of Russia: their distribution, ecophysiological features, multiple utilizations. Eur. J. For. Sci. 13: 25-40. 